\title{
Design of chitosan-alginate core-shell nanoparticules loaded with anacardic acid and cardol for drug delivery
}

\author{
João Campos Paiva Filho', Selene Maia de Morais', Antonio Carlos Nogueira Sobrinho', \\ Gessica Soares Cavalcante ${ }^{1}$, Nilvan Alves da Silva² and Flávia Oliveira Monteiro da Silva Abreu ${ }^{3 *}$
}

\begin{abstract}
'Laboratório de Química de Produtos Naturais, Programa de Pós-graduação em Biotecnologia - RENORBIO, Universidade Estadual do Ceará - UECE, Itaperi Campus, Fortaleza, CE, Brasil

${ }^{2}$ Laboratório de Quimica Analítica e Ambiental, Universidade Estadual do Ceará - UECE, Itaperi Campus, Fortaleza, CE, Brasil

${ }^{3}$ Laboratório de Quimica Analítica e Ambiental, Programa de Pós-graduação em Ciências Naturais - PPGCN, Universidade Estadual do Ceará - UECE, Itaperi Campus, Fortaleza, CE, Brasil

*flavia.monteiro@uece.br
\end{abstract}

\begin{abstract}
Anacardic Acid (AA) and Cardol (CD) are the main constituents of the cashew nut shell liquid, which presented several biological activities. In this study, a $2^{3}$ factorial experimental design was employed in order to evaluate the influence of the reaction conditions in the nanoencapsulation of AA and CD using Chitosan (CH), Alginate (ALG) and Arabic Gum matrices. The nanoparticles (NPs) with higher stability and encapsulation efficiency were those with ALG as an outer coating and with lower content of surfactant. The NPs presented nanometric size with $90 \%$ of the distribution ranging from 70 to $250 \mathrm{~nm}$. The in vitro kinetics revealed that CH-ALG/AA and CH-ALG/CD NPs followed zero-order kinetics model, showing a significantly slow release rate, with values of $33 \%$ and $63 \%$, respectively, after $240 \mathrm{~h}$. Particularly, $\mathrm{CH}-\mathrm{ALG} / \mathrm{CD}$ NPs presented higher inhibitory capacity for all strains of dermatophytes due to their release rate, with promising results for antimicrobial control.
\end{abstract}

Keywords: anacardic acid, cardol, drug delivery, chitosan, nanomaterials.

How to cite: Paiva Filho, J. C., Morais, S. M., Sobrinho, A. C. N., Cavalcante, G. S., Silva, N. A., \& Abreu, F. O. M. S. (2019). Design of chitosan-alginate core-shell nanoparticules loaded with anacardic acid and cardol for drug delivery. Polímeros: Ciência e Tecnologia, 29(4), e2019060. https://doi.org/10.1590/0104-1428.08118

\section{Introduction}

'Anacardic acid and cardol, which are major constituents of the natural cashew nut shell liquid (CNSL), are part of a group of phenolic lipids that has anticancer and antimicrobial activity, among several other activities ${ }^{[1-3]}$. Anacardic acid is a mixture of 6-alkyl salicylic acids and cardol, which is a mixture of alkyl-resorcinols with variations in the unsaturation of their side chain ${ }^{[4]}$. Muroi and Kubo ${ }^{[5]}$ reported antimicrobial activities against Staphylococcus aureus, Streptococus mutans and Helicobacter pylori, also acting in mechanisms that promote the physical rupture of bacterial cell membrane due to their surfactant action ${ }^{[6,7]}$. Anacardic acid displays antimicrobial and anthelmintic uses among others and can be used in large scale; however, it presents a certain instability related to the decarboxylation process, which happens when it is heated. In order to avoid this undesirable reaction, this active compound could be encapsulated using polymer matrixes which could preserve their physical-chemical characteristics. Biopolymers such chitosan and alginate are desirable for encapsulation and has been used for this purpose in the past 20 years ${ }^{[8,9]}$.

According to Peniche and Arguelles-Monal ${ }^{[9]}$ the ability of alginate and chitosan to form polyelectrolyte complexes allows the development of biomaterials, generating tridimensional matrices known as cross-linked gel. Chitosan $(\mathrm{CH})$, a polycation, and alginate, a polyanion, are some examples of polysaccharides used in the preparation of nanoparticles $(\mathrm{NPs})^{[9]}$. In this work, polysaccharide nanoparticles based on chitosan, alginate and arabic gum were prepared with anacardic acid and cardol. $\mathrm{A} 2^{3}$ experiment design was applied in order to optimize the encapsulation efficiency (EE) of the nanoparticles, in vitro release profile and antimicrobial activity was also tested, aiming the production of devices with prolonged antimicrobial action.

\section{Materials and Methods}

\subsection{Materials}

The polymers Chitosan (CH) (Polymar, 72.3\% DD), Alginate (ALG, Dynamic), and Arabic gum (AG, Dynamic) were used. Sodium tripolyphosphate (TPP) (Dynamic) and Tween $80^{\circledR}$ (Dynamic) were also used. Natural CNSL was obtained by maceration of cashew nuts with hexane for one week at room temperature $\left(28^{\circ} \mathrm{C}\right)$. Anacardic acid (AA) and Cardol (CD) were isolated from the natural CNSL by precipitation of AA calcium salt and separation of cardol by solvents extraction ${ }^{[10]}$. 


\subsection{Nanoparticles preparation}

The NPs were prepared according to the following methodology ${ }^{[1]]}$ : A solution containing $50 \mathrm{~mL}$ of $1 \%$ Chitosan and $100 \mathrm{uL}$ of Tween 80 (Sigma-Aldrich ${ }^{\circledR}$ ) were subjected to mechanical stirring. Subsequently, AA or CD was added to the solution and submitted to the ultrasonic bath (Ultra 800, Ciencor Scientific Ltda, São Paulo, Brazil) for 15 min, using Tween 80:AA with a proportion of 1:3 or 1:1. Subsequently, it was added $0.1 \%$ TPP dropwise, using a mass ratio of chitosan:TPP (50:1) and the solution was subjected to magnetic stirring for $30 \mathrm{~min}$. Subsequently $1 \%$ (w/v) ALG or AG gum was added in the solution using a mass ratio chitosan:ALG of 10: 1 ratio and the solution was subjected to magnetic stirring for another $30 \mathrm{~min}$. At last, the solution was centrifuged (Kasvi K14-4000, Curitiba, Brazil) at $4000 \mathrm{rpm}$ for $20 \mathrm{~min}$. The NPs were frozen and lyophilized (L101, Liobras, Brazil).

\subsection{Characterization of NPS}

The reaction yield of NPs production was determined by the ratio of the freeze-dried mass of the NPs regarding the total mass of reagents added. The concentration of compound in the NPs was determined by UV-Vis spectroscopy (Thermo Scientific - Genesys 102S) according to the following procedure: $5 \mathrm{mg}$ of each sample were placed in $5.0 \mathrm{~mL}$ of ethyl alcohol for 48 hours in a hermetic vial. The drug loading was calculated using calibration curves of anacardic acid and cardol at $312 \mathrm{~nm}$ and $275 \mathrm{~nm}$, respectively, as indicated in Equation 1 and Equation 2:

$$
\begin{array}{ll}
y=0.0093 x-0.0209 & R^{2}=0.9999 \\
y=0.0008 x-0.0225 & R^{2}=0.9989
\end{array}
$$

where: $\mathrm{y}$ is the measured absorbance and $\mathrm{x}$ is the concentration of the compound in $\mathrm{mg} / \mathrm{L}$.

The physical-chemical characterization was performed for the optimal formulations of NPs with each encapsulated product, chosen through ANOVA statistical analysis using $\mathrm{p}<0.05$ due to their more satisfactory yield and EE results. Size distribution and Zeta Potential of the NPs were determined in a Nano Zeta Sizer (Malvern 3600). $5 \mathrm{mg}$ of the NPs were placed in $20 \mathrm{ml}$ of deionized water under magnetic stirring for $24 \mathrm{~h}$. Aliquots of $4 \mathrm{ml}$ were withdraw and placed in the cubit for analyses at $\mathrm{pH} 6,0$ at a temperature of $25^{\circ} \mathrm{C}$ in collaboration with the Biomass Technology Laboratory located at the Brazilian Agricultural Research Corporation (Embrapa-CE). The NPs were characterized by FTIR using a Shimadzu Prestige-21 spectrophotometer, where the samples were prepared as potassium bromide pellets $(\mathrm{KBr})$ in the ratio of $1: 20(\mathrm{~m} / \mathrm{m})$ (sample: $\mathrm{KBr})$. The procedure was carried out at the Analytical Center of the Federal University of Rio Grande do Sul (UFRGS).

\subsection{In Vitro release kinetics}

The release kinetics of the AA-loaded and CD-loaded NPs were obtained using a dialysis system. For each sample, $60 \mathrm{mg}$ NPs were introduced into cellulose acetate membranes (14 KDa pore) within a beaker containing $68 \mathrm{~mL}$ of $\mathrm{HCl}$ solution at $\mathrm{pH} 3$. The releasing system was kept under magnetic stirring and constant temperature at $25^{\circ} \mathrm{C}$ for $72 \mathrm{~h}$. Three aliquots were taken at regular time intervals and analyzed by spectrophotometry in the Genesys 10S UV-vis (Thermo Scientific). The concentration of the $\mathrm{AA}$ and $\mathrm{CD}$ present in the medium were determined using Equation 1 and Equation 2.

Kinetic mathematical modeling was applied to verify the mechanism of drug release for the studied formulations. The points obtained from the in vitro release curves were linearized according to the Order-zero, First-order, Higuchi and Hixson-Crowell kinetic model ${ }^{[12]}$. To further elucidate the mechanism of drug release, kinetics based on the Korsmeyer-Peppas model was also evaluated by applying the Equation 3:

$$
\frac{\mathrm{Mt}}{\mathrm{M} \infty}=\mathrm{K} \mathrm{t}^{\mathrm{n}}
$$

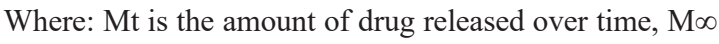
is the amount of drug released as time approaches infinity, $\mathrm{Mt} / \mathrm{M} \infty$ represents the fractional release of the drug, $\mathrm{t}$ is the release time, and $\mathrm{K}$ is the release rate of the system, in which it incorporates structural and geometric characteristics of the polymer system under study.

The diffusion coefficient $\mathrm{n}$ denotes the characteristic of the kinetic mechanism of the drug according to the assumed value. It was calculated the kinetic constants for each model, as well as the exponent $\mathrm{n}$ based on Equation 3 . The correlation coefficient $\left(\mathrm{R}^{2}\right)$ was used as an indication of the best fit for each release system.

\subsection{Antimicrobial activity assay}

The minimum inhibitory concentration (MIC) for Candida spp was determined in accordance with the Clinical and Laboratory Standards Institute ${ }^{[13]}$. The broth microdilution assay for T. rubrum was performed as described on the literature ${ }^{[14,15]}$. The strains were obtained from the fungal collection of the Microbiology Laboratory of the State University Vale do Acaraú, the URM culture collection of the Mycology Department from Federal University of Pernambuco, and Hospital Santa Casa de Misericórdia de Sobral. Four strains of T. rubrum were tested, two of Candida albicans, and two of Candida tropicalis were included in this study. For the broth microdilution method, standardized inocula (2.5-5 x $10^{3} \mathrm{CFU} \mathrm{mL}^{-1}$ for Candida spp. and $5.0 \times 10^{4} \mathrm{CFU} \mathrm{\textrm {mL } ^ { - 1 }}$ for T. rubrum) were prepared by turbidimetry. The suspensions were diluted to 1:2000 for Candida spp. and 1:500 for T. rubrum, both with RPMI 1640 medium (Roswell Park Memorial Institute - 1640) with l-glutamine without sodium bicarbonate (Sigma Chemical Co.). They were buffered to $\mathrm{pH} 7.0$ with $0.165 \mathrm{M}$ MOPS (Sigma Chemical Co.), to obtain inocula of $2.5-5 \times 10^{3} \mathrm{CFU} \mathrm{mL}^{-1}$

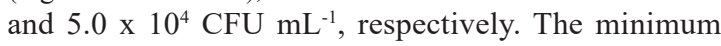
fungicidal concentrations (MFC) for both Candida spp. and T. rubrum were determined as follows: the samples were prepared in 4\% DMSO. Amphotericin B (AMB) and ketoconazole (KTC) (Sigma, Chemical Co., USA) were prepared in distilled water. For the susceptibility analysis, the samples were tested in concentrations ranging from 0.002 to $2.5 \mathrm{mg} / \mathrm{mL}$. The microdilution assay was performed in 96-well microdilution plates and the microplates were 
incubated at $37^{\circ} \mathrm{C}$ and read visually after 2 days for Candida spp. and 5 days for T. rubrum. The MFCs were determined as the lowest concentration resulting in no growth on the subculture after 2 days for Candida spp. and 5 days for T. rubrum ${ }^{[16]}$. All experiments were performed in triplicate.

\subsection{Experimental design}

A factorial experimental design was applied in two levels in order to investigate the effect of three variables on the encapsulation process in the NPs. The studied independent variables were the coating Polymer Matrix (Mat), surfactant dosage (Sd) and type of drug, i.e., Anacardic acid AA and Cardol CD. The dependent variables (responses) were the reaction yield and the drug loading in the NPs. Eight experiments were performed for each dependent variable, grouped into blocks of 2 randomly selected experiments to minimize nuisance effects. The planning matrix containing A, B and C independent variables is shown in Table 1. The statistical treatment of the data was performed through ANOVA analysis in the Excel program (Microsoft 2010). A linear regression analysis was performed among the variables, finding an equation and a $\mathrm{R}^{2}$ value to point out the significance of reaction parameters, $(p)>0.05$.

\section{Results and Discussions}

NPs were produced based on $\mathrm{CH}$ and ALG polysaccharides with a neutral surfactant, in order to provide better solubilization of the compounds in aqueous medium under different reaction conditions. NPs preparation was performed using two distinguished stages. Initially, it was formed a central nucleus with $\mathrm{CH}$ and TPP, using few ionic crosslinking points between the $\mathrm{CH}$ amino groups with the TPP phosphate groups in the presence of surfactant and the active compound. A pre-gel is formed, evidenced by a slight clouding in the solution. After that, an anionic polymer was added into the system. This polymer entangles with the pre-nucleus using strong ionic electrostatic forces as well intermolecular forces, coating the inner core by polyelectrolyte complexation (PEC) [11]. In this study, the proposed structure attempts to entrap higher amount of AA or $\mathrm{CD}$ within the particles and provide a greater protection of these compounds against external environmental factors. Figure 1 shows the representation of the hypothetical structure for the CH-ALG-CD and CH-ALG-AA NPs.
The factorial design and statistical analysis were conducted in order to select NPs with higher drug content and optimized yield, and then those were further investigated regarding in vitro release analysis, as well as characterized by FTIR spectroscopy, particle size, zeta potential and thermogravimetric analysis.

\subsection{Influence of the reaction variables on the yield and drug loading}

The effect of the Polymer Matrix (A), Surfactant dosage (B) and type of drug (C), on the yield and drug loading were evaluated. Table 1 shows the dependent and independent variables. The results showed considerable variation in the reaction yield, with average values of $89 \%$ and $74 \%$ for NPs with AA and CD, respectively. Drug loading also showed a distinct pattern for each drug entrapped, with average values of $26 \%$ and $6 \%$ for NPs loaded with AA and CD, respectively.

ANOVA and linear regression analysis identified that the factor $\mathrm{C}$ was the only factor that influenced on yield, verified by significance analysis. After linear regression, Equation 4 predicts the effect of the variables on yield:

$$
\mathrm{Y} 1=81.25-7.5 \mathrm{C} \quad \mathrm{R}^{2}=0.70
$$

The type of drug entrapped was responsible for $70 \%$ of the yield variation in the experiment, where AA presented a yield higher than $\mathrm{CD}$. The average yield is the intersection

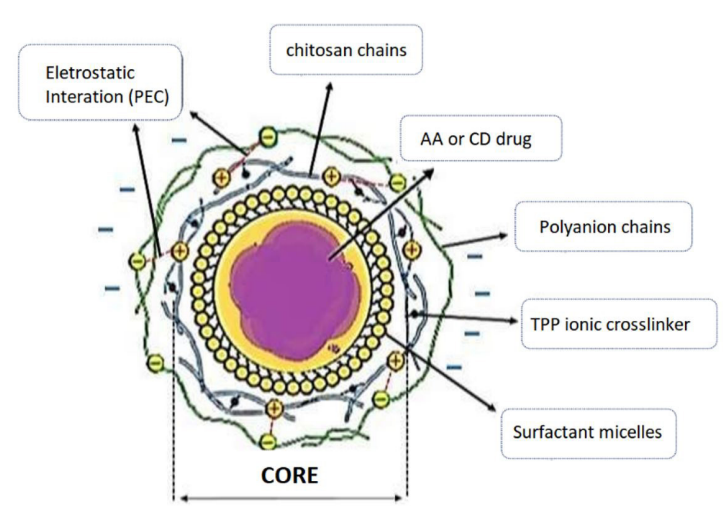

Figure 1. Hypothetical proposed structure of NPs CH-ALG loaded with Cardol (CD) or anacardic acid (AA). (TPP: Tripolyphosphate).

Table 1. $2^{3}$ Factorial design with its drug loading and yield of the material.

\begin{tabular}{|c|c|c|c|c|c|c|}
\hline \multirow{2}{*}{$\mathbf{N}^{0}$} & \multicolumn{4}{|c|}{ Independent variables } & \multirow{2}{*}{ Drug loading } & \multirow{2}{*}{ Yield (\%) } \\
\hline & NP code & Matrix $(A)^{1}$ & Surfactant $(B)^{2}$ & Drug Type $(C)^{3}$ & & \\
\hline 1 & CH-ALG/1sd/AA & -1 & -1 & -1 & $42.6(2.3)$ & 92 \\
\hline 2 & $\mathrm{CH}-\mathrm{AG} / 1 \mathrm{sd} / \mathrm{AA}$ & 1 & -1 & -1 & $38.9(5.7)$ & 89 \\
\hline 3 & CH-ALG/3sd/AA & -1 & +1 & -1 & $12.6(0.7)$ & 85 \\
\hline 4 & $\mathrm{CH}-\mathrm{AG} / 1 \mathrm{sd} / \mathrm{AA}$ & 1 & +1 & -1 & $9.9(0.1)$ & 89 \\
\hline 5 & CH-ALG/1sd/CD & -1 & -1 & +1 & $11.6(0.4)$ & 84 \\
\hline 6 & CH-AG/1sd/CD & 1 & -1 & +1 & $1.5(0.3)$ & 66 \\
\hline 7 & CH-ALG/3sd/CD & -1 & +1 & +1 & $5.6(0.4)$ & 72 \\
\hline 8 & CH-AG/1sd/CD & -1 & +1 & +1 & $5.7(0.4)$ & 73 \\
\hline 9 & CH-ALG/1.3sd/CD & -1 & 0 & +1 & $22.3(0.5)$ & 82 \\
\hline
\end{tabular}

${ }^{1}$ Factor A: Coating Polymer Matrix (Mat), High level (+) = Alginate (ALG); low level (-) = Arabic Gum; ${ }^{2}$ Factor B: Surfactant dosage (Sd), High level $(+)=1: 1$ and low level $(-)=1: 3$; ${ }^{3}$ Factor C: Type of Drug, High level $(+)=$ Cardol and low level $(-)=$ Anacardic Acid. 
value, (81.25). Higher yield was obtained when $\mathrm{C}=-1$, reaching a theoretical yield of 88.75 , close to the values found in reaction number 1 and 2 (NP CH-ALG/1sd/AA and $\mathrm{NP} \mathrm{CH}-\mathrm{AG} / 1 \mathrm{sd} / \mathrm{AA})$. AA and $\mathrm{CD}$ are compounds, which were stabilized in the particle by the surfactant added in the system. However, in an acid medium, AA presents a carboxyl group in the structure, which may interact additionally by ionic forces with available amino groups of $\mathrm{CH}$, becoming even more stable within the particle, and increasing the yield.

Regarding drug loading, the factors $\mathrm{B}, \mathrm{C}$ and the interaction effect $\mathrm{BC}$ were significant verified by ANOVA using $p<0.05$. These factors correspond to the effect of surfactant dosage (B), type of drug (C) and to an interaction effect between surfactant dosage and type of drug (BC). Linear Equation 5 predicts the effect of the variables on the drug loading with $96 \%$ of correlation:

$$
\mathrm{Y} 2=16.1-7.6 \mathrm{~B}-10 \mathrm{C}+7.2 \mathrm{BC} \quad \mathrm{R}^{2}=0,96
$$

Replacing the variables B, C and BC in Equation 5 with normalized values $(-1,+1)$, an optimization was achieved with the following combination of values for $\mathrm{B}, \mathrm{C}$ and $\mathrm{BC}$ respectively: $-1,-1$ and +1 , where the optimal drug loading (Y2) value is $40.8 \%$. This combination matches lower surfactant dosage for anacardic acid (AA) and better interaction among the variables. This theoretical value is also close to the values found in reactions number 1 and 2 (NP CH-ALG/1sd/AA and (NP CH-AG/1sd/AA)).

The drug type is the main factor that directly influences the formed NPs. At a lower level $(\mathrm{C}=-1)$, there was a significant increase on yield and drug loading, regarding the use of AA. In this sense, it is possible to conjecture that due to the acid carboxylic group present only in the chemical structure of AA, strong ionic interactions take place with the amino chitosan groups, in an acid medium, which favors the entrapment into the nanoparticle system. There is also an effect of surfactant and an interaction effect of the surfactant and drug type. When the surfactant dosage is lower $(B=-1)$, regarding the $\mathrm{Sd}: \mathrm{CH}$ 1:3 ratio, in combination with $\mathrm{AA}$ as the drug type $(C=-1)$, best results are obtained (Reaction number 1 and Reaction number 2 , see Table 1 ). This is likely due to these ionic interactions between chitosan and AA, which favored the retention of the drug inside the particle dispensing the use of an excess of surfactant to stabilize the particle. For $\mathrm{CD}$ drug $(\mathrm{C}=+1)$, the surfactant dosage also has to be used at a lower level $(1 \%, \mathrm{~B}=-1)$; however, the optimum theoretical maximum is lower due to negative interaction effect of CD with the surfactant. It seems that the optimum dosage of surfactant to stabilize cardol could be intermediate between the levels studied for this type of drug. Based on these results, a new reaction was produced (reaction number 9 , Table 1 ), using $1.3 \%$ of surfactant dosage, aiming to increase drug loading. Results showed that the drug loading has increased from 11 to $22 \%$ maintaining a high yield, improving significantly the encapsulation system.

In summary, the reaction conditions chosen after statistical analysis were the ones using low surfactant content $(1 \%$ for AA and $1.3 \%$ for $C D$ ). Despite the type of external polymer matrix did not influenced statistically, ALG was chosen as external coating, and the NPs selected were the ones from reaction number $1(\mathrm{CH} / \mathrm{ALG}-\mathrm{AA})$ and reaction number 9 (CH/ALG-CD). Both NPs were evaluated regarding physical-chemical characterization and antimicrobial tests.

\subsection{Infrared spectra}

Figure 2 shows the structural characterization obtained by FTIR spectroscopy for $\mathrm{CH}, \mathrm{ALG}, \mathrm{AA}, \mathrm{CD}$ and NPs loaded with $\mathrm{AA}$ and $\mathrm{CD}$. $\mathrm{CH}$ showed two strong vibrations

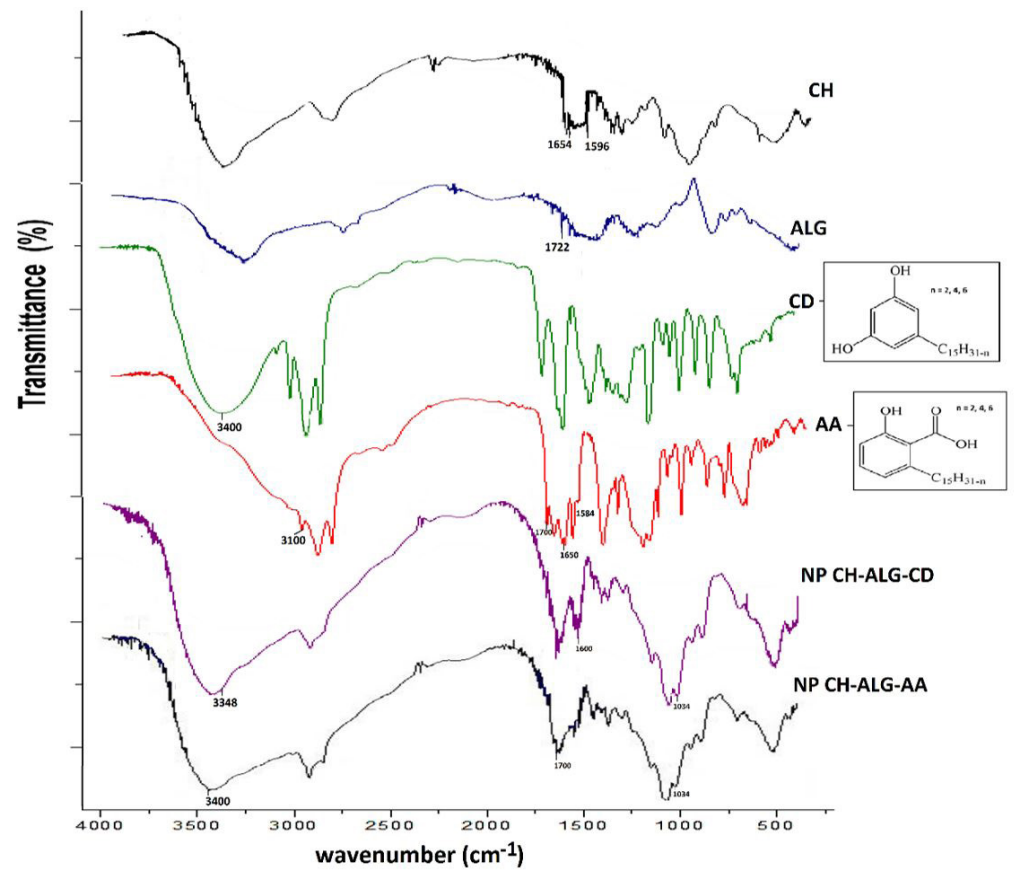

Figure 2. FTIR spectra of the materials Chitosan $(\mathrm{CH})$. sodium alginate (ALG). Cardol (CD). anacardic acid (AA). NPs CH-ALG-CD and NPs CH-ALG-AA. 
at 1654 , assigned to amide I, and at $1596 \mathrm{~cm}^{-1}$ assigned to symmetrical and asymmetrical bending of amine and amide II. ALG has carboxymethyl groups in the structure, with an absorption peak at $1744 \mathrm{~cm}^{-1}$, assigned to the carboxymethyl dimer $(\mathrm{O}=\mathrm{COH} \ldots \mathrm{O}=\mathrm{COH})$ intermolecular interaction which is in agreement with previously published results ${ }^{[17]}$. The anacardic acid exhibits a wide band of axial deformation of the hydroxyl $\mathrm{OH}$ bond (3400-2500 $\left.\mathrm{cm}^{-1}\right)$, referring to the symmetrical stretching of this group. It also presents a band at $1650 \mathrm{~cm}^{-1}$ of the $\mathrm{C}=\mathrm{O}$ stretch of the carboxyl, and also a band at $1584 \mathrm{~cm}^{-1}$ relative to the asymmetrical and symmetrical stretching of the -COO groups. On the other hand, cardol exhibits axial deformation of the hydroxyl $\mathrm{OH}$ bond $\left(3400 \mathrm{~cm}^{-1}\right)^{[18]}$.

The NPs showed the axial deformation of OH $\left(3400 \mathrm{~cm}^{-1}\right)$, $\mathrm{C}-\mathrm{O}-\mathrm{C}\left(1034 \mathrm{~cm}^{-1}\right)$ and $=\mathrm{C}-\mathrm{H}$ of the benzene ring in either cardol or anacardic acid $\left(1610-1550 \mathrm{~cm}^{-1}\right)$. The vibrations of the charged amino groups were observed near $3448 \mathrm{~cm}^{-1}$ after formation of the complex, meaning that the - $\mathrm{COO}^{-}$ions of the ALG bonded with $-\mathrm{NH}_{3}^{+}$of the $\mathrm{CH}$ forming the polyelectrolyte complex and modifying the vibrational modes of the polysaccharides ${ }^{[17,19]}$. Even with the overlapping of the vibrational modes of the drugs with those of the $\mathrm{CH}$ and ALG, it is possible to observe, in the spectrum of NPs with AA, the increase in intensity around $1700 \mathrm{~cm}^{-1}$, and it indicates the presence of the carboxylic acid groups of AA incorporation of the drug incorporated into the NPs.

\subsection{Particle size and zeta potential}

The particle size was evaluated for CH-ALG NPs loaded with AA and CD. Figure 3 shows the particle size profile for the NPs, where the distribution pattern presented some variations according to the type of active encapsulated principle.

NPs loaded with AA presented an average particle size of $220 \mathrm{~nm}$, where $90 \%$ of the presented values are ranging from 90 to $250 \mathrm{~nm}$, with a unimodal distribution. NPs loaded with CD presented a discrete bimodal profile, where $95 \%$ of the NPs presented a size from 70 to $200 \mathrm{~nm}$, and 5\% of the NPs presented higher particle size, with values from $250 \mathrm{~nm}$ to $530 \mathrm{~nm}$. Both systems presented a high fraction of particles below $250 \mathrm{~nm}$, which are favorable applications for use in in vivo release systems ${ }^{[20-22]}$. According to the literature, particles with this particle size are susceptible to a higher rate of mobility in controlled release system applications compared to larger materials ${ }^{[21,22]}$.

The SEM micrographs of ALG-CHI hydrogels loaded with AA and CD are shown in supplementary information ${ }^{[23-26]}$ (Figure S1). The morphology showed micro spheroid-shaped clusters, formed by the agglomeration of several spherical NPs. This morphology is in according to the literature ${ }^{[27]}$ where aggregation of spherical NPs often is observed as a result of freeze-drying process. NPs loaded with CD (Figure $\mathrm{S} 1 \mathrm{~b}$ ) presented higher particle size than those loaded with AA (Figure S1 a).

The Zeta Potential was investigated for the NPs in aqueous suspension at $\mathrm{pH}$ 6.0. NPs presented negative values of surface potential, respectively of -18.8 and $-9.8 \mathrm{mV}$ for those loaded with AA and CD. The NPs loaded with AA presented a negative charge density higher than those loaded with $\mathrm{CD}$, being comparatively more stable and less predisposed to agglomerate, as observed in SEM micrographs. The negative zeta potential values detected are attributed to the ion charge (-COO) of the alginate on the outer surface of the particle and indicate that the $\mathrm{CH}$-based core was successfully coated ${ }^{[28,29]}$. Therefore, NPs CH-ALG assumed moderate values of potential, with over $90 \%$ of the fraction with size lower than $250 \mathrm{~nm}$ (Figure $3 \mathrm{a}$ and $3 \mathrm{~b}$ ), with good probability to disperse successfully in future in vivo applications.

\subsection{In Vitro release profiles}

The in vitro release profile of the CH-ALG NPs, performed at $\mathrm{pH} 3.0$ to simulate the stomach acid condition, is displayed in Figure 4. It evidenced lower release rates in the first 48 hours, with corresponding release values of $10 \%$ and $6 \%$ for $\mathrm{CD}$ and $\mathrm{AA}$, and even after $96 \mathrm{~h}$, NPs release was increased to $19 \%$ and $7 \%$ for $\mathrm{CD}$ and $\mathrm{AA}$, respectively. After $240 \mathrm{~h}, \mathrm{CH}-\mathrm{ALG}-\mathrm{AANPs}$ released only $33 \%$ of AA, showing a strong interaction to the surfactant-CH-ALG system, which prevented its releasing. In acid medium, the carboxyl groups present in the AA acid formed strong ionic and hydrogen intermolecular interactions with amine $\mathrm{CH}$ protonated groups, and the hydrocarbon chain portion of the AA were (a)

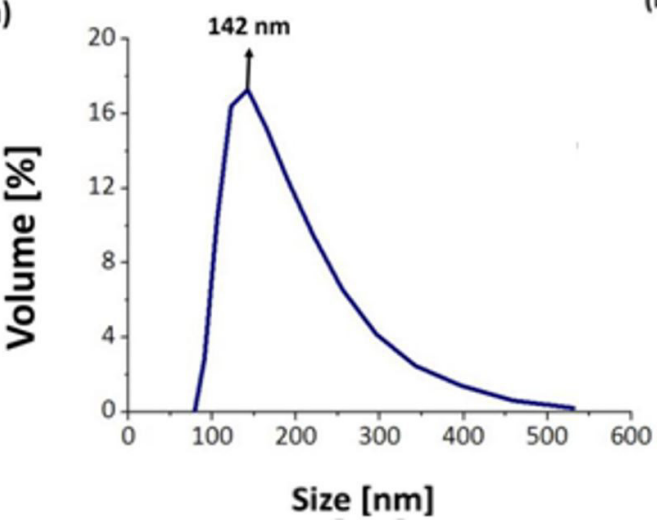

(b)

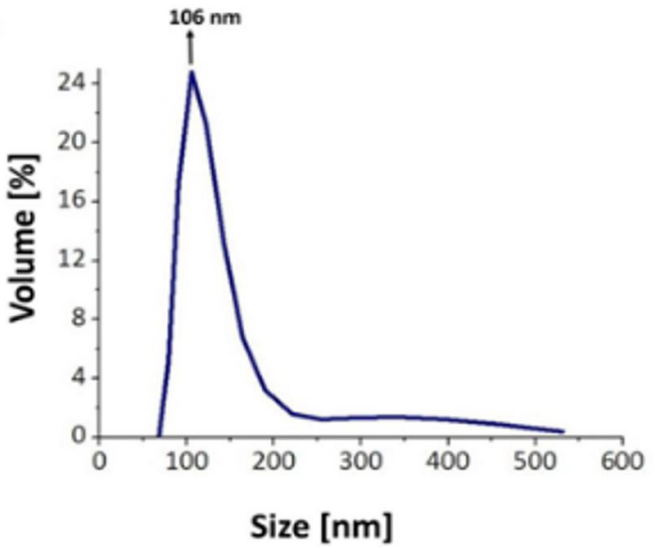

Figure 3. Distribution of particle size based on volume for (a) CH-ALG-AA NPs and (b) CH-ALG-CD NPs. 
successfully stabilized by the surfactant. In this case, AA was retained inside the NP in acid medium for a prolonged time (over 10 days). On the other hand, CH-ALG-CD NPs presented a satisfactory controlled release profile. Table 2 shows the kinetic constant and the correlation coefficient for the studied Kinetic models. A kinetic study based on the conventional release models ${ }^{[12]}$ from the in vitro release of $\mathrm{AA}$ and $\mathrm{CD}$ revealed that both systems were best fitted in the zero-order kinetic model, due to their higher correlation coefficient $\left(\mathrm{R}^{2}\right)$. This model is based on the slow release of active substances from systems that gradually disintegrate in the dissolution medium ${ }^{[30]}$. In this case, the abrupt increase in the release profile after $180 \mathrm{~h}$ is attributed to the slowly dissolution of the NP particle, where the active drug was dispersed in the aqueous system. These NPs are intended to serve active substances in a controlled and prolonged way, as is the case with some of the transdermal systems, such as matrix tablets, coated forms, osmotic systems, drugs with low solubility, among others ${ }^{[29]}$.

In our study, the release occurred only after a considerably prolonged period $(240 \mathrm{~h})$, and there was a maximum release of approximately $63 \% \mathrm{CD}$ and $39 \% \mathrm{AA}$, in accordance with zero-order kinetic model. In this system, NPs were produced using $\mathrm{CH}$ and ALG as coatings; a possible explanation for this behavior can be associated to a two-step mechanism: initially, occurs the migration of the active principle by diffusion from the inner core to the interface of $\mathrm{CH}-\mathrm{ALG}$ coating, with possible accumulation of a retention volume ${ }^{[29]}$. A concentration gradient is produced and the release rate is slow and controlled due to the affinity of AA and CD by the $\mathrm{CH}-\mathrm{ALG}$ interfaces. Over time, is reached a saturation concentration in the interface, followed by the rupture of $\mathrm{CH}-\mathrm{ALG}$ barrier, causing a sudden increase in the release medium $^{[26]}$.

A similar pattern is found in the literature ${ }^{[31]}$. Alginatecalcium chloride microparticles loaded with eucalyptus oil presented a slow release rate, with $60 \%$ of eucalyptus oil release after $97 \mathrm{~h}$. Such a sustained released improve the product shelf life due to the protective effect of the nanoparticles.

The Korsmeyer-Peppas model provides a constant $\left(\mathrm{K}_{\mathrm{KP}}\right)$, regarding the release rate, where $\mathrm{CD}$ presented a release rate $\left(\mathrm{K}_{\mathrm{KP}}\right)$ higher than AA, with values of respectively 4.15 and $2.87 \mathrm{~h}^{-1}$. Both systems presented diffusion coefficients outside the limits of the Korsmeyer-Peppas model $(n<0.5)$. Reports in the literature suggest that a classical Fickian diffusion or "Less Fickian" behavior is characterized when the values of the diffusion coefficient are below the proposed limit ${ }^{[32,33]}$. A similar pattern was also found in other studies, where values below 0.5 were reported in Lippia sidoides release from ALG and Cashew gum $\mathrm{NPs}^{[34]}$ and also ibuprofen release from polysaccharide matrices based on hydroxypropyl meticellulose ${ }^{[35]}$.

\subsection{Antimicrobial activity}

The encapsulation of natural products with previously characterized biological action is important in order to improve physical-chemical conditions and bioavailability, optimizing a controlled release. Alginate and chitosan are among the most widely polysaccharides in synthesis of nanoparticles. As they were prepared in aqueous medium

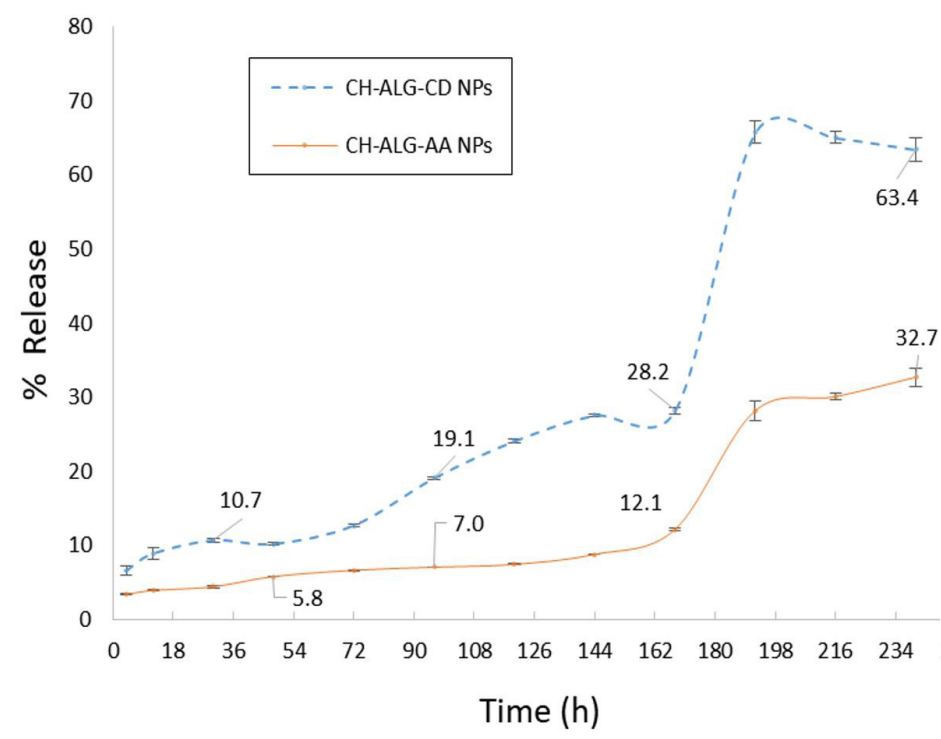

Figure 4. Controlled in vitro release of Nanoparticles with anacardic acid and cardol.

Table 2. Kinetic constant $(\mathrm{K})$ and the correlation coefficient $\left(\mathrm{R}^{2}\right)$ for the Kinetic models studied: zero order, first-order, Higuchi, HixsonCrowell and Korsmeyer-Peppas

\begin{tabular}{|c|c|c|c|c|c|c|c|c|c|c|c|}
\hline \multirow{2}{*}{ NPs } & \multicolumn{2}{|c|}{ Zero-order } & \multicolumn{2}{|c|}{ First-order } & \multicolumn{2}{|c|}{ Higuchi } & \multicolumn{2}{|c|}{ Hixson-Crowell } & \multicolumn{3}{|c|}{ Korsmeyer-Peppas } \\
\hline & $\mathbf{R}^{2}$ & $\mathbf{K}_{0}\left(\mathbf{h}^{-1}\right)$ & $\mathbf{R}^{2}$ & $K_{1}\left(h^{-1}\right)$ & $\mathbf{R}^{2}$ & $\mathbf{K}_{\mathrm{H}}\left(\mathbf{h}^{-1 / 2}\right)$ & $\mathbf{R}^{2}$ & $\mathbf{K}_{\mathrm{HC}}\left(\mathbf{h}^{-1 / 3}\right)$ & $\mathbf{R}^{2}$ & $\mathbf{K}_{\mathrm{KP}}\left(\mathbf{h}^{-\mathrm{n}}\right)$ & $\mathbf{n}$ \\
\hline CH-ALG-AA & 0.9441 & 0.0560 & 0.9389 & 0.0007 & 0.9035 & 0.7594 & 0.9408 & 0.0009 & 0.9081 & 2.8734 & 0.2563 \\
\hline CH-ALG-CD & 0.9786 & 0.1392 & 0.9641 & 0.0007 & 0.8950 & 1.8879 & 0.9657 & 0.0024 & 0.8195 & 4.1514 & 0.3194 \\
\hline
\end{tabular}


Table 3. Minimum inhibitory concentration (MIC) and minimum fungicidal concentration (MFC).

\begin{tabular}{lcccccc}
\hline \multirow{2}{*}{ Strains } & \multicolumn{3}{c}{ Minimum Inhibitory Concentration/Minimum Fungicidal Concentration (mg/mL) } \\
\cline { 2 - 7 } & AA & NP/AA & CD & NP/CD & AMB $^{1}$ & KTC $^{\mathbf{2}}$ \\
\hline C. albicans LABMIC 0105 & $1.25 / 2.5$ & NI & NI & NI & 0.125 & NT \\
C. albicans LABMIC 0407 & NI & NI & NI & NI & 0.125 & NT \\
C. tropicalis LABMIC 0110 & $1.25 / 2.5$ & NI & NI & NI & 0.125 & NT \\
C. tropicalis LABMIC 0111 & $2.5 / 2.5$ & $2.5 / 2.5$ & $2.5 / 2.5$ & $2.5 / 2.5$ & 0.125 & NT \\
T. rubrum LABMIC 5906 & $0.075 / 0.039$ & $0.625 / 0.312$ & $0.625 / 0.312$ & $0.625 / 0.312$ & NT & 0.25 \\
T. rubrum LABMIC 6205 & $0.075 / 0.039$ & $0.625 / 0.312$ & $0.625 / 0.312$ & $0.625 / 0.312$ & NT & 0.25 \\
T. rubrum LABMIC 6213 & $0.075 / 0.039$ & $0.625 / 0.312$ & $0.625 / 0.312$ & $0.625 / 0.312$ & NT & 0.25 \\
T. rubrum LABMIC 6753 & $0.075 / 0.039$ & $0.625 / 0.312$ & $1.25 / 0.625$ & $0.625 / 0.312$ & NT & 0.25 \\
\hline AA: Ant
\end{tabular}

AA: Anacardic Acid; NP/AA: Nanoparticles CH-ALG-AA; CD: Cardol ; NP/CD: Nanoparticles CH-ALG-CD; NI: no inhibition; NT: not tested; ${ }^{1} \mathrm{AMB}$ : standard Amphotericin B; ${ }^{2} \mathrm{KTC}$ : standard Ketoconaloze.

without the presence of harmful substances, it is considered a safe method, without the presence of harmful substances ${ }^{[36]}$. Regarding the in vitro antifungal activity of AA and $\mathrm{CD}$ in its free and encapsulated forms, the results are summarized in Table 3. The compounds showed no significant activity when tested against strains of Candida spp (C. albicans LABMIC 0105, C. albicans LABMIC 0407, C. tropicalis LABMIC 0110 and C. tropicalis LABMIC 0111), except for anacardic acid, which was active in inhibiting the microbial growth of $C$. albicans LABMIC 0105 . However, the results showed MIC and MFC values from 0.625 and $0.312 \mathrm{mg} / \mathrm{mL}$, respectively, for all strains of dermatophytes (T. rubrum LABMIC 5906, T. rubrum LABMIC 6205, T. rubrum LABMIC 6212 and T. rubrum LABMIC 6753).

The fungistatic (MIC) and fungicidal (MFC) action against dermatophyte strains showed that $\mathrm{AA}$ and $\mathrm{CD}$, through an action on the fungal cell membrane, possibly inhibited the growth of the microorganisms in a dose-dependent concentration. Dermatophytes such as Trichophyton rubrum with a high affinity for keratinizined tissues are fungi responsible for dermatophytosis of human and veterinary skin infections ${ }^{[37]}$. The results demonstrate that the encapsulation of AA and CD decreased the MIC and MFC values, expressing the antifungal activity because it requires lower samples concentration to perform the same activity when compared to the non-encapsulated samples. In the antifungal assays, the incubation period of the sample with the dermatophytic fungi is at least 120 hours, and then the controlled release of the encapsulated samples allowed an antimicrobial action by inhibiting growth at lower concentrations than for the free samples.

$\mathrm{AA}$ and $\mathrm{CD}$ are phenolic compounds present in Cashew nut shell liquid with antimicrobial action against Gram-positive and negative bacteria strains proven by previous studies ${ }^{[1,35]}$. The antimicrobial action seems to be related to the amphipathic character of the phenolic lipids. The interaction of the hydroxyl groups of the aromatic ring with phospholipids by means of hydrogen bonds and the lipophilic side chain are characteristics responsible for the high affinity of the CNSL to the lipid bilayers present in the bacterial membranes ${ }^{[38]}$. AA and CD are entrapped within the NPs. However, based on the in vitro release profile, it is expected that CH-ALG-CD NPs present higher antimicrobial efficiency, due to the progressive release of $\mathrm{CD}$ over the $120 \mathrm{~h}$ test. Evidently, both systems presented high content of the retained compound after the test period, with a potential effect of microbial inhibition in a period of over 10 days. This structural feature contributes to the greater antimicrobial activity of the encapsulated samples.

\section{Conclusions}

Chitosan-alginate NPs can be used for encapsulation of cardol and anacardic acid using a two stage procedure of gelification followed by external coating. Statistical analysis showed that the characteristics of the NPs are strongly dependent on the surfactant dosage and the type of compound encapsulated. Low surfactant dosage is required to obtain with higher yield and higher loading. The negative zeta potential values suggested that the external coating of the particles was indeed composed of anionic ALG chains. The kinetic study showed a desirable profile, best fitted in zero-order model, probably through a two-step mechanism, caused by accumulation of the active principles in CH-ALG interface by migration followed by the rupture of the interfacial barrier after saturation, with a steep increase in the release medium.

Microbiological studies showed that $\mathrm{CH}-\mathrm{ALG}-\mathrm{CD}$ maintained the same moderate activity of free $\mathrm{CD}$ when compared to the standard, showing effectiveness in the encapsulation system.

\section{Acknowledgements}

The authors thank Professor Dr. Men de Sá from Embrapa Agroindustria Tropical for zeta potential and particle size analysis. This work was supported by the Conselho Nacional de Desenvolvimento Científico - CNPq [Projeto Universal 442965/2014-1].

\section{References}

1. Hamad, F. B., \& Mubofu, E. B. (2015). Potential biological applications of bio-based anacardic acids and their derivatives. International Journal of Molecular Sciences, 16(4), 8569-8590. http://dx.doi.org/10.3390/ijms16048569. PMid:25894225.

2. Kubo, I., Masuoka, N., Ha, T. J., \& Tsujimoto, K. (2006). Antioxidant activity of anacardic acids. Food Chemistry, 99(3), 555-562. http://dx.doi.org/10.1016/j.foodchem.2005.08.023.

3. Kamath, V., \& Rajini, P. S. (2007). The efficacy of cashew nut (Anacardium occidentale L.) skin extract as a free radical scavenger. Food Chemistry, 103(2), 428-433. http://dx.doi. org/10.1016/j.foodchem.2006.07.031. 
4. Correia, S. J., David, J. P., \& David, J. M. (2006). Metabolitos secundários de espécies de anacardiaceae. Quimica Nova, 29(6), 1287-1300. http://dx.doi.org/10.1590/S0100-40422006000600026.

5. Muroi, H., \& Kubo, I. (1996). Antibacterial activity of anacardic acid and totarol, alone and in combination with methicillin, against methicillin-resistant Staphylococcus aureus. Journal of Applied Microbiology, 80(4), 387-394. http://dx.doi. org/10.1111/j.1365-2672.1996.tb03233.x. PMid:8849640.

6. Kubo, I., Muroi, H., \& Kubo, A. (1994). Naturally occurring antiacne agents. Journal of Natural Products, 57(1), 9-17. http://dx.doi.org/10.1021/np50103a002. PMid:8158169.

7. Green, I. R., Tocoli, F. E., Lee, S. H., Nihei, K., \& Kubo, I. (2008). Design and evaluation of anacardic acid derivatives as anticavity agents. European Journal of Medicinal Chemistry, 43(6), 1315-1320. http://dx.doi.org/10.1016/j.ejmech.2007.08.012. PMid:17959274.

8. Gharsallaoui, A., Roudaut, G., Chambin, O., Voilley, A., \& Saurel, R. (2007). Applications of spray-drying in microencapsulation of food ingredients: an overview. Food Research International, 40(9), 1107-1121. http://dx.doi.org/10.1016/j.foodres.2007.07.004.

9. Peniche, C., \& Arguelles-Monal, W. (2001). Chitosan based polyelectrolyte complexes. Macromolecular Symposia, 168(1), 103-116. http://dx.doi.org/10.1002/15213900(200103)168:1<103::AID-MASY103>3.0.CO;2-K.

10. Paramashivappa, R., Kumar, P. P., Vithayathil, P. J., \& Rao, A. S. (2001). Novel method for isolation of major phenolic constituents from cashew (anacardium occidentale L.) nut shell liquid. Journal of Agricultural and Food Chemistry, 49(5), 25482551. http://dx.doi.org/10.1021/jf001222j. PMid:11368634.

11. Abreu, F. O. M. S., Silva, N. A., Sipauba, M. S., Pires, T. F. M., Bomfim, T. A., Monteiro, O. A. C., Jr., \& Forte, M. M. C. (2018). Chitosan and gum arabic nanoparticles for heavy metal adsorption. Polímeros: Ciência e Tecnologia, 28(3), 231-238. http://dx.doi.org/10.1590/0104-1428.02317.

12. Dash, S., Murthy, P. N., Nath, L., \& Chowdhury, P. (2010). Kinetic modeling on drug release from controlled drug delivery systems. Acta Poloniae Pharmaceutica Drug Research, 64(3), 217-223. PMid:20524422.

13. Clinical and Laboratory Standards Institute. (2008). Reference method for broth dilution antifungal susceptibility testing of yeasts (approved standard. Document M27. CLSI) (3rd ed., Vol. M27-A3). Wayne: Clinical and Laboratory Standards Institute.

14. Sobrinho, A. C. N., Souza, E. B., Rocha, M. F. G., Albuquerque, M. R. J. R., Bandera, P. N., Santos, H. S., Cavalcante, C. S. P., Oliveira, S. S., Aragão, P. R., Morais, S. M., \& Fontenelle, R. O. S. (2016). Chemical composition, antioxidant, antifungal and hemolytic activities of essential oil from baccharis trinervis (lam.) pers. (asteraceae). Industrial Crops and Products, 84, 108-115. http://dx.doi.org/10.1016/j.indcrop.2016.01.051.

15. Clinical and Laboratory Standards Institute. (2008). Reference method for broth dilution antifungal susceptibility testing of filamentous fungi (approved Standard. Document M38. CLSI) (2nd ed., Vol. M38-A2). Wayne: Clinical and Laboratory Standards Institute.

16. Fontenelle, R. O. S., Morais, S. M., Brito, E. H. S., Brilhante, R. S. N., Cordeiro, R. A., Nascimento, N. R. F., Kerntopf, M. R., Sidrim, J. J. C., \& Rocha, M. F. G. (2008). Antifungal activity of essential oils of Croton species from the Brazilian Caatinga biome. Journal of Applied Microbiology, 104(5), 1383-1390. http:// dx.doi.org/10.1111/j.1365-2672.2007.03707.x. PMid:18298533.

17. Dubey, R., Bajpai, J., \& Bajpai, A. K. (2016). Chitosan-alginate nanoparticles (CANPs) as potential nanosorbent for removal of $\mathrm{Hg}(\mathrm{II})$ ions. Environmental Nanotechnology, Monitoring \& Management, 6, 32-44. http://dx.doi.org/10.1016/j. enmm.2016.06.008.
18. Rodrigues, F. H. A., Feitosa, J. P. A., Ricardo, N. M. P. S., França, F. C. F., \& Carioca, J. O. B. (2006). Antioxidant activity of cashew nut shell liquid (CNSL) derivatives on the thermal oxidation of syntheticcis-1,4-polyisoprene. Journal of the Brazilian Chemical Society, 17(2), 265-271. http://dx.doi. org/10.1590/S0103-50532006000200008.

19. Paula, H. C. B., Sombra, F. M., Cavalcante, R. F., Abreu, F. O. M. S., \& de Paula, R. C. M. (2011). Preparation and characterization of chitosan/cashew gum beads loaded with Lippia sidoides essential oil. Materials Science and Engineering C, 31(2), 173-178. http://dx.doi.org/10.1016/j.msec.2010.08.013.

20. Abreu, F. O. M. S., Paula, H. C. B., Oliveira, E. F., \& Paula, R. C. M. (2012). Chitosan/cashew gum nanogels for essential oil encapsulation. Carbohydrate Polymers, 89(4), 1277-1282. http:// dx.doi.org/10.1016/j.carbpol.2012.04.048. PMid:24750942.

21. Fazil, M., Md, S., Haque, S., Kumar, M., Baboota, S., Sahni, J., \& Ali, J. (2012). Development and evaluation of rivastigmine loaded chitosan nanoparticles for brain targeting. European Journal of Pharmaceutical Sciences, 47(1), 6-15. http://dx.doi. org/10.1016/j.ejps.2012.04.013. PMid:22561106.

22. Ridolfi, D. M., Marcato, P. D., Justo, G. Z., Cordi, L., Machado, D., \& Duran, N. (2012). Chitosan-solid lipid nanoparticles as carriers for topical delivery of tretinoin. Colloids and Surfaces. B, Biointerfaces, 93, 36-40. http://dx.doi.org/10.1016/j. colsurfb.2011.11.051. PMid:22244299.

23. Rodrigues, F. H. A., Feitosa, J. P. A., Ricardo, N. M. P. S., França, F. C. F., \& Carioca, J. O. B. (2006). Antioxidant activity of cashew nut shell liquid (CNSL) derivatives on the thermal oxidation of syntheticcis-1,4-polyisoprene. Journal of the Brazilian Chemical Society, 17(2), 265-271. http://dx.doi. org/10.1590/S0103-50532006000200008.

24. Ribeiro, V. G. P., Barreto, A. C. H., Denardin, J. C., Mele, G., Carbone, L., Mazzetto, S. E., Sousa, E. M. B., \& Fechine, P. B. A. (2013). Magnetic nanoparticles coated with anacardic acid derived from cashew nut shell liquid. Journal of Materials Science, 48(22), 7875-7882. http://dx.doi.org/10.1007/s10853013-7477-4.

25. Eldin, M. S. M., Hashem, A. E., Tamer, T. M., Omer, A. M., Yossuf, M. E., \& Sabet, M. M. (2017). Development of cross linked chitosan/alginate polyelectrolyte proton exchanger membranes for fuel cell applications. International Journal of Electrochemical Science, 12, 3840-3858. http://dx.doi. org/10.20964/2017.05.45.

26. Kittur, F. S., Harish Prashanth, K. V., Udaya Sankar, K., \& Tharanathan, R. N. (2002). Characterization of chitin, chitosan and their carboxymethil derivatives by differential scanning calorimetry. Carbohydrate Polymers, 49(2), 185-193. http:// dx.doi.org/10.1016/S0144-8617(01)00320-4.

27. Ghadi, A., Mahjoub, S., Tabandeh, F., \& Talebnia, F. (2014). Synthesis and optimization of chitosan nanoparticles: potential applications in nanomedicine and biomedical engineering. Caspian Journal of Internal Medicine, 5(3), 156-161. PMid:25202443.

28. Lertsutthiwong, P., Rojsitthisak, P., \& Nimmannit, U. (2009). Preparation of turmeric oil-loaded chitosan-alginate biopolymeric nanocapsules. Materials Science and Engineering C, 29(3), 856-860. http://dx.doi.org/10.1016/j.msec.2008.08.004.

29. Parmar, A., \& Sharma, S. (2010). Engineering design and mechanistic mathematical models: standpoint on cutting edge drug delivery. Trends in Analytical Chemistry, 100, 15-35. http://dx.doi.org/10.1016/j.trac.2017.12.008.

30. Varelas, C. G., Dixon, D. G., \& Steiner, C. A. (1995). Zero-order release from biphasic polymer hydrogles. Journal of Controlled Release, 34(3), 185-192. http://dx.doi.org/10.1016/01683659(94)00085-9.

31. Noppakundilograt, S., Piboon, P., Graisuwan, W., Nuisin, R., \& Kiatkamjornwong, S. (2015). Encapsulation eucalyptus 
oil in ionically cross-linked alginate microcapsules and its controlled release. Carbohydrate Polymers, 131, 23-3. http:// dx.doi.org/10.1016/j.carbpol.2015.05.054. PMid:26256156.

32. Asare-Addo, K., Levina, M., Rajabi-Siahboomi, A. R., \& Nokhodchi, A. (2010). Study of dissolution hydrodynamic conditions versus drug release from hypromellose matrices: the influence of agitation sequence. Colloids and Surfaces. B, Biointerfaces, 81(2), 452-460. http://dx.doi.org/10.1016/j. colsurfb.2010.07.040. PMid:20729043.

33. Wang, J., Wu, W., \& Lin, Z. (2008). Kinetics and thermodynamics of the water sorption of 2-hydroxyethyl methacrylate/styrene copolymer hydrogels. Journal of Applied Polymer Science, 109(5), 3018-3023. http://dx.doi.org/10.1002/app.28403.

34. Oliveira, E. F., Paula, H. C. B., \& Paula, R. C. M. (2014). Alginate/cashew gum nanoparticles for essential oil encapsulation. Colloids and Surfaces. B, Biointerfaces, 113(1), 146-151. http:// dx.doi.org/10.1016/j.colsurfb.2013.08.038. PMid:24077112.

35. Shoaib, M. H., Tazeen, J., Merchant, H. A., \& Yousuf, R. I. (2006). Evaluation of drug release kinetics from ibuprofen matrix tablets using HPMC. Pakistan Journal of Pharmaceutical Sciences, 19(2), 119-124. PMid:16751122.
36. Bouchara, J. P., Mignon, B., \& Chaturvedi, V. (2017). Dermatophytes and dermatophytoses: A thematic overview of state of the art, and the directions for future research and developments. Mycopathologia, 182(1-2), 1-4. http://dx.doi org/10.1007/s11046-017-0114-z. PMid:28138872.

37. Parasa, L. S., Tumati, S. R., Kumar, L. C. A., Chigurupati, S. P., \& Rao, G. S. (2011). In vitro-antimicrobial activity of cashew (Anacardium occidentale, L.) nuts shell liquid against methicillin resistant Stephylococcus aureus (MRSA) clinical isolates. International Journal of Pharmacy and Pharmaceutical Sciences, 3(4), 436-440. Retrieved in 2019, April 1, from https://innovareacademics.in/journal/ijpps/ Vol3Issue4/2724.pdf

38. Kozubek, A., \& Tyman, J. H. P. (1999). Resorcinolic lipids, the natural non-isoprenoid phenolic amphiphiles and their biological activity. Chemical Reviews, 99(1), 1-26. http:// dx.doi.org/10.1021/cr970464o. PMid:11848979.

Received: Apr. 01, 2019

Revised: Feb. 17, 2020

Accepted: Feb. 19, 2020 


\section{Supplementary Material}

Supplementary material accompanies this paper.

Figure S1: SEM micrographs of (a) NPs CH-ALG-AA and (b) NPs CH-ALG-CD.

Figure S2: Thermograms of (a) AA and (b) NPs CH-ALG-AA; (c) CD and (d) NPs CH-ALG-CD. Green Line: (\%) weight change as function of the temperature; blue line: Derivative weight change as a function of increasing temperature

This material is available as part of the online article from http://www.scielo.br/po 